\title{
Excellent Radiological Response with Modern Contemporary Proton Beam Therapy in Favorable Molecular Low-Intermediate Grade Oligodendroglioma: A Report of Two Cases
}

\author{
Raees Tonse $^{1}$ MP Noufal ${ }^{2}$ Dayananda Shamurailatpam² ${ }^{2}$ Rakesh Jalali ${ }^{1}$ \\ ${ }^{1}$ Department of Radiation Oncology, Apollo Proton Cancer Centre, \\ Chennai, Tamil Nadu, India \\ 2Department of Medical Physics, Apollo Proton Cancer Centre, \\ Chennai, Tamil Nadu, India

\begin{abstract}
Address for correspondence Rakesh Jalali, MD, Department of Radiation, Oncology, Apollo Proton, Cancer Centre, 4/661, Dr. Vikram Sarabai Instronic, Estate 7th Street, Dr. Vasi, Estate, Phase II, Tharamani, Chennai, Tamil Nadu 600 096, India
\end{abstract} \\ (e-mail: rjalali@apollohospitals.com).
}

Ind J Med Paediatr Oncol 2021;42:110-112.

\begin{abstract}
Radiotherapy (RT) has been a long-standing treatment option for low-grade glioma. Improvements in tumor control and reduced radiation-related toxicity can be

Keywords attributed to advances in neuroimaging as well as RT treatment planning and delivery

- low-grade glioma techniques. The molecular markers such as isocitrate dehydrogenase and Ipl9q play a

$-\mathrm{IDH}$ key role in determining which patients will benefit most from combined radiation and

- oligodendroglioma

- proton beam therapy

$-\mathrm{PCV}$ systemic therapy. We hereby report two cases of favorable molecular low-intermediate grade oligodendroglioma treated with modern proton pencil-beam therapy under high-precision image guidance showing excellent radiological response that is usually not seen with conventional photon radiation.
\end{abstract}

\section{Introduction}

Low-grade glioma generally refers to the two most common histologic subtypes of the World Health Organization (WHO) grade II gliomas: diffuse astrocytoma and oligodendroglioma (ODG). ${ }^{1}$ They have a protracted natural history, which in most instances, terminates with transformation into high-grade gliomas. Treatment options for these tumors have ranged from observation to a biopsy for histological confirmation to aggressive radical resections followed by adjuvant radiotherapy (RT) with or without additional chemotherapy. ${ }^{2}$ Based on the increasing knowledge and emerging data, 2016 WHO brain tumor classification uses molecular information to establish a brain tumor diagnoses. Gliomas with isocitrate dehydrogenase (IDH) 1 and 2 mutation and lp/19q co-deletion with the histological pattern of ODGs form the

DOI https://doi.org/ 10.1055/s-0041-1729342 ISSN 0971-5851 most favorable prognosis. ${ }^{3}$ Modern highly conformal RT techniques have been shown to minimize RT doses to the normal brain significantly as compared with conventional RT, without compromising RT doses essential for durable tumor control. However, the recent RT techniques have mitigated several of the long-term challenges, issues such as neurocognitive decline continue to exist. ${ }^{4.5}$ Proton beam therapy (PBT) has emerged peerless in terms of precision, safety, and efficacy due to its unique physical characteristics of minimal exit dose that has an unequivocal dosimetric superiority over high-end photon/standard X-ray beam therapy and is particularly advantageous in low-intermediate grade gliomas because of their very high probability of long-term cures. ${ }^{6}$ We would like to report our preliminary experience of two cases of favorable low-intermediate grade ODG treated at our facility, showing excellent radiological response. (c) 2021. Indian Society of Medical and Paediatric Oncology

This is an open access article published by Thieme under the terms of the Creative Commons Attribution-NonDerivative-NonCommercial-License, permitting copying and reproduction so long as the original work is given appropriate credit. Contents may not be used for commercial purposes, or adapted, remixed, transformed or built upon. (https://creativecommons.org/licenses/by-nc-nd/4.0/).

Thieme Medical and Scientific Publishers Pvt. Ltd. A-12, 2nd Floor, Sector 2, Noida-201301 UP, India 


\section{Case Reports}

\section{Case 1}

A 45-year-old male was evaluated for seizures in September 2018. His magnetic resonance imaging (MRI) showed a diffuse lesion in the right medial temporal, right occipital, and posterior parietal lobe involving the splenium of the corpus callosum. He underwent right parieto-occipital navigation-guided burhole biopsy of the occipital lobe lesion in November 2018. The histopathology report was suggestive of infiltrative glioma grade IL. The molecular markers were IDH mutation, lp19q codeletion, and telomerase reverse transcriptase mutation that were positive. Because of age $>40$ years and some features of a high-grade component on radiology, he was advised concurrent radiation with PBT to dose $54 \mathrm{GyE}$ in 30 fractions at $1.8 \mathrm{GyE}$ per fraction along with

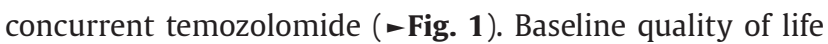
(QOL) questionnaire was assessed; he tolerated treatment well with no interruptions. Response assessment scan after 4 weeks showed a very good response, with a nearly $50 \%$ reduction in the disease burden ( - Fig. 2 A).

\section{Case 2}

A 31-year-old male was evaluated for generalized tonicclonic seizures in 2011. His MRI brain revealed a large hyperintense area in the anterior occipital and post-temporal lobe. He underwent left temporoparietal craniotomy and resection of the lesion. Histopathology was reported as ODG WHO grade II (lpl9q-codeleted). He was kept on a regular follow-up with 6 months MRI. Serial imaging revealed a gradual increase in the size of the left temporal lobe lesion. He also had occasional episodes of generalized tonic-clonic seizure. The last MRI brain in July 2018 showed infiltrative lesion

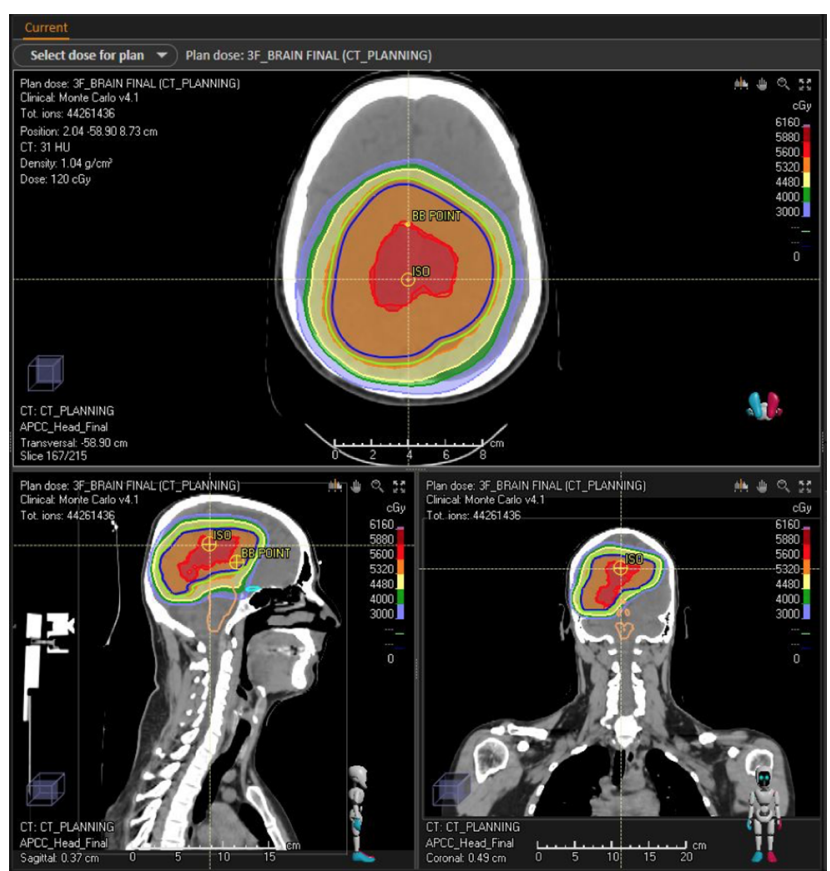

Fig. 1 Representative images of proton pencil-beam therapy plan showing dose-wash in axial, sagittal, and coronal view. Planning is based on Monte Carlo optimization algorithms.

measuring $5 \times 3.8 \times 5.56 \mathrm{~cm}$ in the left posterior temporal lobe with ill-defined patchy enhancement consistent with disease progression. His case was discussed in the neuro-oncology tumor board meeting. Due to young age group and favorable histology, it was decided to offer him adjuvant PBT to a dose of 55.8 GyE in 31 fractions at $1.8 \mathrm{GyE}$ per fraction along with concurrent temozolomide. Baseline QOL questionnaire was assessed; he tolerated treatment well with no interruptions. Response assessment scan after 4 weeks showed a very good response, with a nearly $50 \%$ reduction in the disease burden (-Fig. 2B).

\section{Discussion}

The management of low-grade gliomas is an evolving and often controversial topic. With a long-term survival rate that exceeds $90 \%$, therapy selection involves careful consideration of minimizing late toxicity from the surgery, chemotherapy, and irradiation. Surgery, radiation therapy, and chemotherapy may be used as individual therapies or in combination, offering different therapeutic response depending on the clinical setting. IDH mutation has a predictive value for response to chemotherapy in gliomas. Extended follow-up of a large randomized controlled trial (RTOG 9402) that compared procarbazine/lomustine/vincristine (PCV) chemotherapy in combination with RT to RT alone demonstrated a survival advantage of the combination in IDH-mutant anaplastic gliomas. ${ }^{7}$ Alkylating chemotherapy using the

\section{Figure 2A}

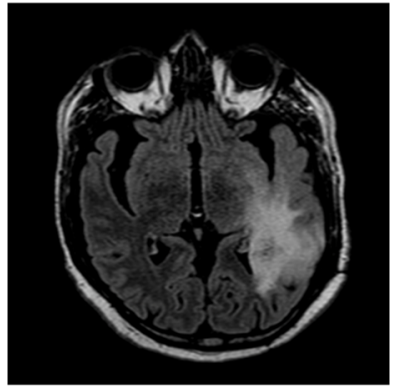

Figure 2B

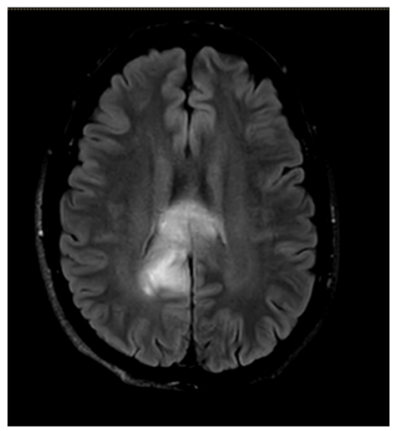

Fig. 2 (A) Axial fluid-attenuated inversion-recovery image pre(image on the left) and post-proton beam therapy (image on the right) of a case of low-grade glioma showing a very good response to proton beam therapy; (B) axial fluid-attenuated inversion-recovery image pre- (image on the left) and post-proton beam therapy (image on the right) of a case of low-grade glioma showing an excellent response. 
PCV regimen initially and temozolomide more recently have assumed a firm place in the treatment of patients with low-grade gliomas. ${ }^{8}$

Prospective and retrospective studies indicate that radiation therapy offers effective, long-term disease control, but perceptions of radiation toxicity have produced limited enthusiasm surrounding its use, particularly in younger adults., ${ }^{6,9,10}$ Several studies have attempted to reduce the effect of late radiation toxicity by using advanced radiation techniques to reduce the dose administered to normal tissues.and by the use of advanced radiation techniques. ${ }^{10}$ PBT, by the modern pencil beam technique with image guidance, is particularly promising because it allows for reductions in the low and intermediate radiation dose to normal tissue outside of the target volume, which is shown by various dosimetric studies. ${ }^{9}$ Radiation side effects such as neurocognitive impairment, neurologic deficits, neurovascular compromise, neuroendocrine deficiency, and second malignancies can be significantly reduced by reducing the radiation dose to critical organs such as the hippocampus, cochlea, and hypothalamic-pituitary axis. A Phase II Randomized Trial of Proton versus Photon Therapy (IMRT) for cognitive preservation in patients with IDH mutant, low-to-intermediate grade gliomas (NRG-BN005) is currently ongoing and should give us better insights in the future.

We report our preliminary experience of two cases of favorable low-intermediate grade glioma treated with modern contemporary PBT showing excellent radiological response. Neurocognitive assessment, QOL, and response assessment are prospectively captured and will be published once we have a longer follow-up.

\section{Conclusion}

PBT due to its unprecedented precision for more focused targeting of tumors, less damage to healthy tissue around the tumor and lesser chance of intellectual impairment, vascular complications, and secondary cancers can be the treatment of choice in low-intermediate grade glioma and is likely to emerge as the standard of care in the management of these tumors.

\section{Disclaimer}

The authors certify that they have obtained all appropriate patient consent forms. In the form, the patient(s) has/have given his/her/their consent for his/her/their images and other clinical information to be reported in the journal. The patients understand that their names and initials will not be published and due efforts will be made to conceal their identity, but anonymity cannot be guaranteed.

\section{Financial Support and Sponsorship}

Nil.

\section{Conflicts of Interest}

There are no conflicts of interest.

\section{Acknowledgment}

We thank our colleagues from neurosurgery, radiation oncology, and medical physics for patient referrals and treatment planning.

\section{References}

1 Louis DN, Perry A, Reifenberger G, et al. The 2016 World Health Organization Classification of Tumors of the Central Nervous System: a summary. Acta Neuropathol 2016;131(6):803-820

2 Pignatti F, van den Bent M, Curran D, et al. European Organization for Research and Treatment of Cancer Brain Tumor Cooperative GroupEuropean Organization for Research and Treatment of Cancer Radiotherapy Cooperative Group. Prognostic factors for survival in adult patients with cerebral low-grade glioma. J Clin Oncol 2002;20(8):2076-2084

3 Santosh V, Sravya P, Gupta T, et al. ISNO consensus guidelines for practical adaptation of the WHO 2016 classification of adult diffuse gliomas. Neurol India 2019;67(1):173-182

4 Armstrong GT, Conklin HM, Huang S, et al. Survival and longterm health and cognitive outcomes after low-grade glioma. Neuro-oncol 2011;13(2):223-234

5 Gondi V, Hermann BP, Mehta MP, Tomé WA. Hippocampal dosimetry predicts neurocognitive function impairment after fractionated stereotactic radiotherapy for benign or low-grade adult brain tumors. Int J Radiat Oncol Biol Phys 2012;83(4):e487-e493

6 Hauswald H, Rieken S, Ecker S, et al. First experiences in treatment of low-grade glioma grade I and II with proton therapy. Radiat Oncol 2012;7:189

7 Buckner JC, Shaw EG, Pugh SL, et al. Radiation plus procarbazine, CCNU, and vincristine in low-grade Glioma. N Engl J Med 2016;374(14):1344-1355

8 van den Bent MJ, Baumert B, Erridge SC, et al. Interim results from the CATNON trial (EORTC study 26053-22054) of treatment with concurrent and adjuvant temozolomide for $1 \mathrm{p} / 19 \mathrm{q}$ non-co-deleted anaplastic glioma: a phase 3, randomised, open-label intergroup study. Lancet 2017;390(10103): 1645-1653

9 Thurin E, Nyström PW, Smits A, et al. Proton therapy for lowgrade gliomas in adults: a systematic review. Clin Neurol Neurosurg 2018;174:233-238

10 Grosshans DR, Mohan R, Gondi V, Shih HA, Mahajan A, Brown PD. The role of image-guided intensity modulated proton therapy in glioma. Neuro-oncol 2017;19(suppl_2): ii30-ii37 